\title{
APPLYING LAYERED GRAPHS TO EVIDENCE CENTERED DESIGN BASED SERIOUS GAMES ASSESSMENT
}

\author{
Jan K. Argasiński and Iwona Grabska-Gradzińska \\ Department of Games Technology, Faculty of Physics, Astronomy and Applied Computer Science, \\ Jagiellonian University in Krakow, Poland
}

\begin{abstract}
In this paper authors propose idea for inclusion of the Evidence Centered-Design (ECD) driven assessments into graph- based scenarios/levels generating system. The concept is to position ECD's evidences as elements of layered graphs representing game state and possible player's/student's actions. Graph's series of productions can be then compared to task assigned to player/student and evaluated. This mechanism combines one of the most popular methods of designing educational assessments with procedural generation of scenarios/levels and with core ideas derived from game design - such as concept of mechanics, represented here as the game design patterns.
\end{abstract}

\section{KEYWORDS}

Educational Assessment, Serious Games, Mechanics, Layered Graphs, Game Design Patterns, Evidence Centered Design

\section{INTRODUCTION - KEY CONCEPTS}

\subsection{The Problem of Knowledge Representation and Open Assessment}

Today serious games are becoming one of the most promising areas in computer-mediated education (Zhonggen, 2019). There are two main reasons behind that fact. First - the generation for whom playing games is one of the most important ways of consuming culture has long since entered schools (what is more - teachers are also increasingly people "brought up on games"). Second - games are more immersive, interactive, social objects than textbooks - which significantly influences the involvement of students in the teaching material (Almeida \& Simoes, 2019).

Two main problems when it comes to serious games design are 1) methods of representation and transfer of knowledge appropriate for the medium and 2) ways to evaluate the educational progress of students in dynamic and open virtual environment. The goal of this paper is to outline possible solution to both these problems by applying the game design patterns and layered graphs for serious gameplay generation and assessment's evidences creation.

\subsection{Key Concept: Educational Assessment and Evidence-Centered Design}

Educational assessment is the process of systematic review of learning outcomes and progress using empirical data. Frequently, evaluation is framed into the canvas of knowledge, skill, abilities (KSA), and sometimes also beliefs. To achieve proper assessment there is a need for structured data on learning process and its outcomes. The simplest method in educational appraisal is testing. Nowadays, however, the methodology of stealth assessment is increasingly valued. The idea is that evaluation should be "seamlessly woven directly into the fabric of the instructional environment to support learning of important content and key competences. This represents quiet, yet powerful process by which learner performance data are continuously gathered during playing/learning (...)" (Shute, 2011, p. 504). 
Evidence-Centered Design (ECD) is the framework for the design and implementation of assessments that provides systematic overview of evidences of educational progress (Arieli-Attali et al., 2019). It was introduced by Mislevy et al. (1994) and developed by many other scholars (Phelps et al., 2020; Johnson et al., 2018; Hao \& Mislevy, 2018). The core idea is to provide system of models that describe various parts of educational endeavor. The ECD contains of the Students Model, the Evidence Model, the Task Model, the Assembly Model, and the Presentation Model (Mislevy et al., 2003). For us the most important is the Student Model which defines variables in relation to measured KSAs, and the Evidence Model which provide instructions on how to update the Student Model's variables in the light of work products from assigned tasks. Main mechanism of evaluation is hidden in the "identification and summary of evidence within tasks, in terms of observable variables" (Mislevy et al., 2003, p. 9). Variables are used for storing and updating the information about the student's proficiency in particular domains.

\subsection{Key Concept: Patterns in (Serious) Games Design}

Assessment in the ECD is performed based on evidences gathered from tasks assigned to student. In the realm of serious games tasks translate to game mechanics defined after Miguel Sicart (2008) as "methods invoked by agents to interact with the game state". Mechanics are means in which player (student) interacts with objects in environment to perform tasks. Outcomes from this interaction can be interpreted as evidences of knowledge, skill, ability of belief.

There are various definitions of mechanics, and there are various ways to systematize them (see: Sicart, 2008). Because all of them are essentially repetitive - they can be catalogued into patterns. The advantage of such approach is the ability to arrange mechanics into sequences and the possibility of automatic evaluation (balancing). Among the most interesting ways to systematize mechanics are propositions by Björk \& Holopainen (2005) and Adams \& Dormans (2012).

\subsection{Key Concept: Layered Graphs in Serious Games Content Representation}

Layered graphs are widely used in the fields, where data structures represent complex dependencies of real world, and where data manipulation and problem solving can be translated into well-defined graph algorithms (e.g. shortest path finding, BFS, DFS, etc.). It can be useful in serious games for e.g., user's behavior or multioptional choices analysis (Grabska-Gradzińska et al., 2012). Graph systems are also used in the design of support systems. (Vermeulen et al., 2016)

By graph model it is understood the system of nodes as information containers and edges as the representation of dependencies among the nodes. The layers help with assigning specific values or features to specific groups of nodes, which supports the flexibility of representation, and universality of structure. (Haahtela et al., 2015).

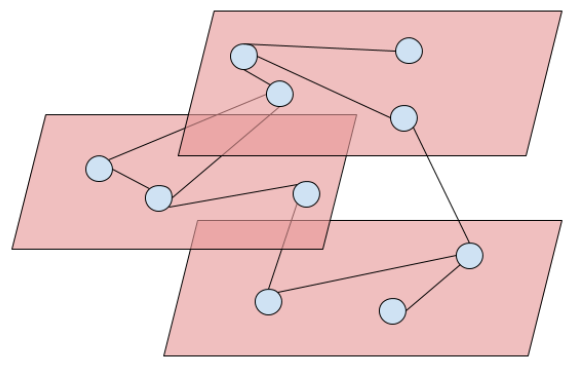

Figure 1. The idea of the layered graph: nodes (blue dots) gathered into three groups (red shapes) connected within one layer and between the layers (black lines)

The graph model, which represents the game state changes after every players' action. For the sake of formalization all these actions are mapped by the graph operations called "productions". It is the formal mechanism to connect player's behavior with its impact on the state of the game world. 


\section{PROPOSED SOLUTION - GRAPH GENERATED CONTENT}

In this proposal the ECD's Task Model, generalized game mechanics (patterns) and in game world objects are mapped into graph model elements. Every node stands for the possible player's action. The graph's edges starting from first node represent the available choices of operations. Every node directly connected means the next possible operation. Some of nodes are collected in the Evidence Layer, which means, that they are crucial for player's evaluation. The path in the gameplay's graph represents the sequence of player's behaviors. The edges between nodes ensure that the cause and the effect relationship between succeeding actions is preserved. The assigned task is represented as subgraph with one initial node from any layer and group of nodes from the Evidence Layer, which collects player's responses to initial stimuli.

\section{SIMPLE CASE STUDY: OSH SERIOUS GAME}

\subsection{The OSH Game Design Requirements}

The Occupational Safety and Health (OSH) games are the specific category of serious games, because the procedures collected in the safety instruction manuals give small chance for spontaneity, but, depending on the circumstances, every single schema can be mixed with others or performed in different order. The evaluation of the course of the OSH games is not a trivial problem also because of the gravity of the matter. That being said - there are many ways to formalize player's behavior e.g., locating direct testing questions in the game narrative (Grabska-Gradzińska, 2016), using gameplay patterns (Argasiński \& Grabska-Gradzińska, 2017), hazard control matrix (Greuter et al., 2013), etc. Another aspect of evaluation of the player knowledge is the idea of collecting additional information of player's physical reactions during the gameplay, e.g. time gap between question and replay, eye-tracking analysis, affective indicators of engagement etc. In every case the goal is to systematically assess changes in player's and game's states as evidences of some particular knowledge, skill or ability.

\subsection{Patterns in OSH Game Design}

For every serious game deigned in this framework there is a need for creation of an extensive, closed list of meaningful interaction that can occur during the gameplay. There is of course very large body of possible actions performed by player in the gaming environment but for purposes of educational assessment we need only catalogue of that which are in the first order 1) predictable (probable), and in the second 2) related to evaluation (linked to the tasks) or not.

For the purpose of this paper closed list of patterns with detailed description was not created due to lack of space - instead ad hoc and quite obvious mechanics (such as "reaching the doors" and "closing the windows") were used.

\subsection{Graph Generated Content in OSH Serious Game}

Let's take into consideration player's/student's task/goal based on fire safety instructions of one of the public institutions:

Task: Evacuation.

Initial production: Fire alarm (1)

Productions: Collecting personal belongings (2); Turning off electric devices (3); Closing the windows (4); Face covering (5); Location changing (6-11,13,14); Reaching the doors (7); Using the elevator (12); Reaching the meeting point (15). 
Evidences: Finding the shortest path to the meeting point (subgraph: 6-13-14-15); Closing the doors while leaving the room (subgraph: 6); Turning off electric devices (subgraph 3); Closing the windows (subgraph: 4); Face covering [in case of fumes] (subgraph: 5); Using the elevator [negative evidence] (subgraph 12).

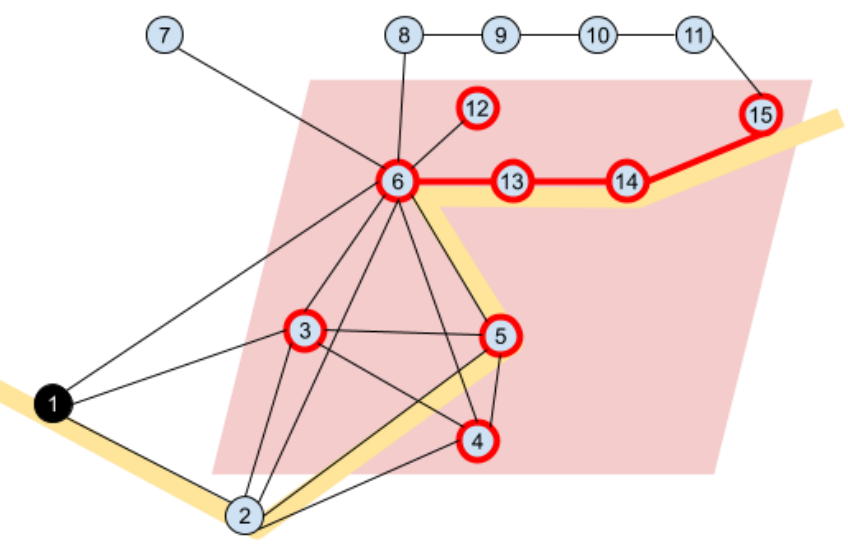

Figure 2. The game graph of the task "Escape": evidences (red subgraphs), other productions (blue dots), initial production (black dot), player's path (yellow line)

\subsection{The Assessment}

In the provided simple example there is the graph that describes possible player's actions that map to the ECD's evidences of the OSH knowledge, skills and abilities. Note that given proper boundary conditions and extensive catalogue of patterns various graphs can be generated for the purpose of similar assessments. The graphs can than serve as input to procedural content generating systems (such as level builders) to ensure that student's KSAs are assessed in multiple similar but different gameplays. This is the main feature of proposed solution.

\section{CONCLUSION}

In the above paper the outline of method of generating assessments for the serious games was provided. In the future follow up of the concept various detailed formalizations for generating graphs could be provided. There is also potentially interesting topic of using sophisticated statistical models (such as Bayesian networks) in order to generate the series of gameplay-based assessment. These topics, however interesting, are beyond the scope of the above paper - nonetheless they show the possibilities contained in the simple idea of combining the ECD, game design patterns and layered graphs.

\section{REFERENCES}

Adams, E. \& Dormans, J., 2012. Game Mechanics. Advanced Game Design. New Riders, San Francisco, USA.

Almeida, F. et al., 2019. The Role of Serious Games, Gamification and Industry 4.0 Tools in the Education 4.0 Paradigm. Contemporary Educational Technology, 10, 120-136, 10.30935/cet.554469.

Argasiński, J. K., Grabska-Gradzińska, I., 2017. Patterns in serious game design and evaluation application of eye-tracker and biosensors. ICAISC 2017. LNCS (LNAI), vol. 10246, pp. 367-377. Springer, Cham

Arieli-Attali, M. et al., 2019. The Expanded Evidence-Centered Design (e-ECD) for Learning and Assessment Systems: A Framework for Incorporating Learning Goals and Processes Within Assessment Design. Frontiers in Psychology, 10, 10.3389/fpsyg.2019.00853.

Björk, S. \& Holopainen, J., 2005. Patterns in Game Design. Charles River Media, Newton, USA. 
Grabska-Gradzińska, I. et al., 2012. Towards a graph-based model of computer games. Advances in Information Technologies and Communication 2012: proceedings of Joint International Conferences on ICT, Computer Science Series, ISSN 2213-2805, 3.

Grabska-Gradzińska, I., 2015. How to predict behavior at risk: educational game story analysis using layered graphs and eyetracking system. Proceedings of the 23rd International Workshop on Intelligent Computing in Engineering: EG-ICE 2016, Kraków, Poland.

Greuter, S. et al., 2013. Designing a game for occupational health and safety in the construction industry. $10.1145 / 2336727.2336740$.

Haahtela P. et al., 2015. Gamification of Education: Cities Skylines as an Educational Tool for Real Estate and Land Use Planning Studies

Hao, J. \& Mislevy, R., 2018. The Evidence Trace File: A Data Structure for Virtual Performance Assessments Informed by Data Analytics and Evidence-Centered Design: The Evidence Trace File. ETS Research Report Series, $10.1002 /$ ets2.12215.

Johnson, E. et al., 2018. Using evidence centered design to create a special education teacher observation system. Educational Measurement Issues and Practice, 37, 10.1111/emip.12182.

Mislevy, R. et al., 2003. A Brief Introduction to Evidence-Centered Design. CSE Report 632. US Department of Education, 10.1002/j.2333-8504.2003.tb01908.x.

Mislevy, R. J., 1994. Evidence and inference in educational assessment. Psychometrika, 59, 439-483.

Phelps, G., 2020. Developing Assessments of Content Knowledge for Teaching Using Evidence-centered Design. Educational Assessment, 10.1080/10627197.2020.1756256.

Shute, V., 2011. Stealth Assessment in computer-based games to support learning. Computer Games and Instruction, 55. Sicart, M., 2008. Defining Game Mechanics. Game Studies. The International Journal of Computer Game Research, 8.

Vermeulen M. et al., 2016. DISCO, a Formal Model of Serious Games to Help Teachers at the Design Stage. Doctoral Consortium CSEDU 2016 (DCCSEDU 2016), Apr 2016, Rome, Italy.

Yu, Z., 2019. A Meta-Analysis of Use of Serious Games in Education over a Decade. International Journal of Computer Games Technology, 1-8, 10.1155/2019/4797032. 